In der Rubrik „Literatur kompakt" werden die wichtigsten Originalarbeiten aus der internationalen Fachliteratur referiert.

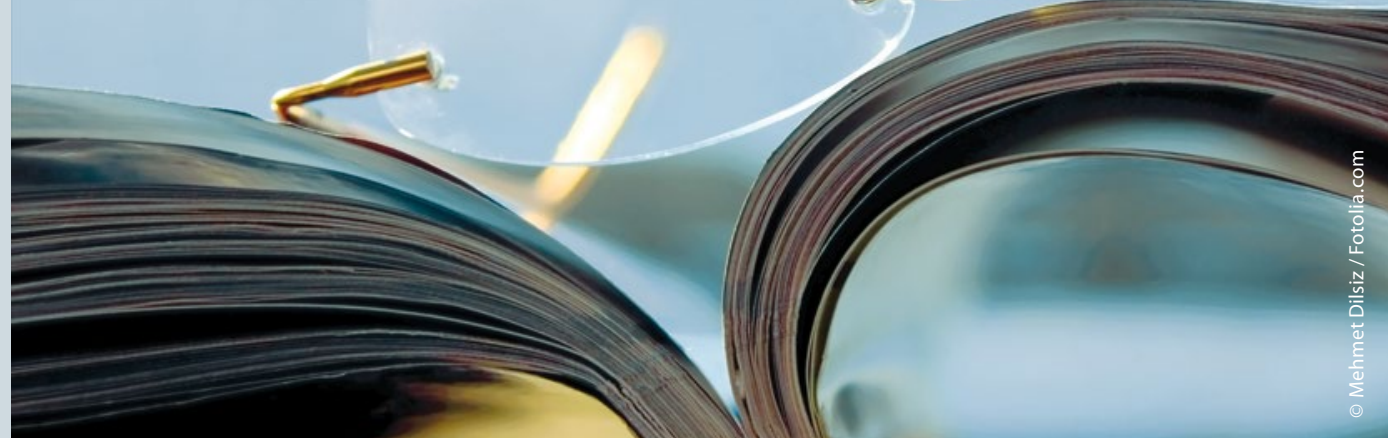

\section{Strategie der Aktiven Überwachung geht auf}

\section{Die Aktive Überwachung von Patienten mit lokal begrenztem Prostatakarzi- nom lohnt sich: In einer US-Studie ist damit innerhalb von zehn Jahren $62 \%$ der Betroffenen eine Krebstherapie erspart geblieben.}

$\mathrm{D}$ ie frühe Diagnose eines Prostatakarzinoms (PCa) ist heute nicht mehr automatisch der Startschuss, um eine Therapie zu beginnen. Mit der Strategie der Aktiven Überwachung (Active Surveillance, AS) kann vielen Männern mit lokal begrenztem PCa eine Krebsbehandlung erspart bleiben. Wie häufig im Zeitraum während der Aktiven Überwachung bei Patienten mit PCa tatsächlich eine Therapie nötig wird, haben Dr. Mark Preston und Kollegen vom Brigham and Women's Hospital, Boston, USA, in einer Kohortenstudie untersucht. Grundlage für die Studie bildeten die Daten von 469 Männern, die zwischen 1997 und 2009 mit einem lokalisierten PCa diagnostiziert worden waren und die sich für eine Strategie der Aktiven Überwachung entschieden hatten.

Die zum Zeitpunkt der Diagnose im Schnitt 68-jährigen Patienten wurden im Median 4,8 Jahre beobachtet. Bei $94 \%$ der Patienten lag der PSA-Wert zum Zeitpunkt der Diagnose $<10$ ng/ml (Median $5,1 \mathrm{ng} / \mathrm{ml})$. Der Gleason-Score erreichte bei $98,2 \%$ einen Wert von $\leq 6$, bei $1,7 \%$ einen Wert von $3+4=7$. Beim Staging wurden $94 \%$ der Patienten der Gruppe T1c, 6 \% der Gruppe T2a zugeordnet.

Nach fünf Jahren Beobachtungszeit wurden $77 \%$ der Studienteilnehmer noch nicht behandelt, nach zehn Jahren lag die Quote der Therapiefreien bei $62 \%$. Nach

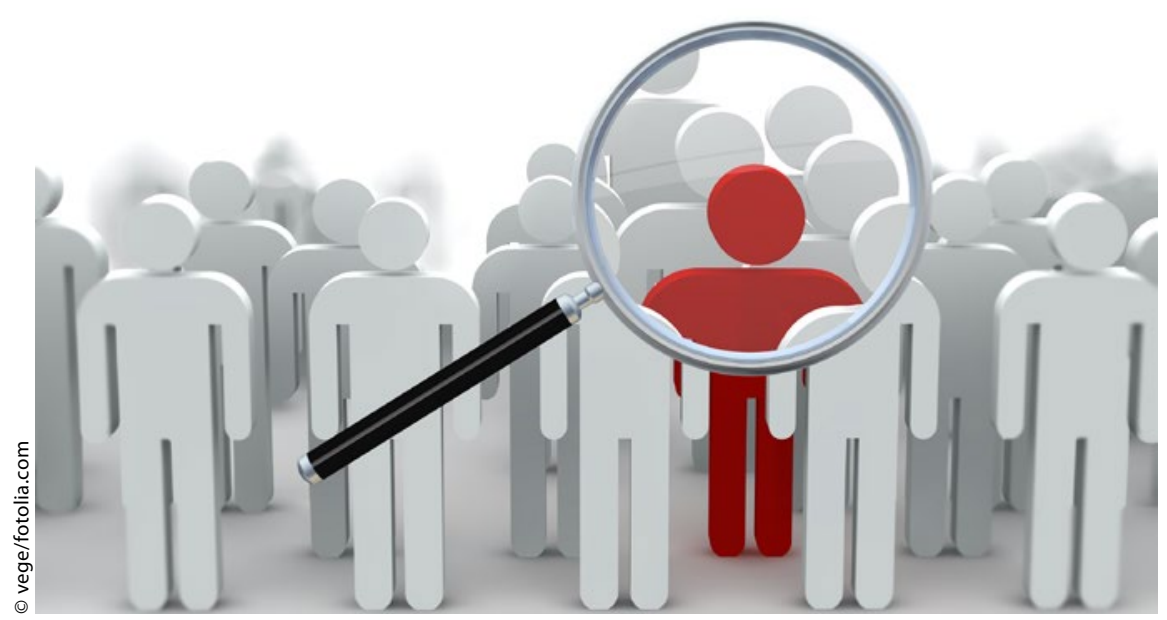

Aktives Überwachen lohnt sich bei den meisten Männern mit Low-Risk-Prostatakarzinom. durchschnittlich 1,94 Jahren unterzogen sich $65,7 \%$ der Männer mindestens einer erneuten Biopsie.

Gründe für den Entschluss zur Therapie während der Aktiven Überwachung waren eine schlechtere Klassifikation bei der Kontrolle (45\%), eine PSA-Progression (30\%), der Patientenwunsch (12\%), eine Progression bei digital-rektaler Untersuchung (5\%) sowie Metastasenbildung (4\%). Von den behandelten Patienten erhielten $50 \%$ eine Radiotherapie, $22 \%$ unterzogen sich einer radikalen Prostatektomie, bei $15 \%$ erfolgte eine Brachytherapie und $12 \%$ entschlossen sich zur antiandrogenen Therapie. Keiner der Patienten starb innerhalb von zehn Jahren an seinem PCa. Das metastasenfreie Überleben lag nach fünf Jahren bei 99 und nach zehn Jahren bei $97 \%$, das Gesamtüberleben bei 95 und $88 \%$.

Fazit: Mit der Strategie der Aktiven Überwachung war es möglich, dass die meisten Männern mit Low-Risk-Prostatakarzinom, auf eine Krebstherapie verzichten konnten. Eine wichtige Komponente der Aktiven Überwachung ist die Kontrollbiopsie: Ein höherer GleasonScore oder eine Progression des Tumorvolumens können den Wendepunkt hin zur aktiven Therapie darstellen. Die Daten der Studie, so betonten Preston und Kollegen, unterstrichen die Sicherheit der Methode bei sorgfältiger Patientenselektion.

Dr. Christine Starostzik

Preston MA, Feldman AS, Coen JJ et al. Active surveillance for low-risk prostate cancer: need for intervention and survival at 10 years. Urol Oncol. 2015; pii:S1078-1439(15)00194-5. 